\title{
Diabetic ketoacidosis; Annual Incidence and Precipitating Factors at King Abdulaziz Medical city, Jeddah
}

\author{
Shahd Alahmadi ${ }^{1}$, Aliya Ragaban ${ }^{2}$, Shahd Alblowi ${ }^{2}$, Entisar Aljumail ${ }^{3}$, Hawazen Zarif ${ }^{4}$ \\ ${ }^{1}$ Ibn Sina National College for Medical Studies, ${ }^{2}$ King Abdulaziz University Hospital,3 Qassim University,4 King \\ Saud University for Health Science
}

\begin{abstract}
Introduction: Diabetes is a recognizable and major health burden in Saudi Arabia. The incidence of Diabetes Mellitus Type 1 (DM1) reached 109.5 per 100,000 child and adolescent. One of the major life-threatening complications of DM1 is Diabetic Ketoacidosis (DKA). This study aims to estimate DKA incidence among DM1 patients and possible risk factors.

Methods: Retrospective review for DM1 patients at King Abdul-Aziz Medical City, and who was presented with DKA during the past six and half years.

Results: Three hundred and fifty DM1 patients were included and (72) experienced DKA during study period. The average annual DKA onset was $(6.2 \%)$. Mean age was $(22.82 \pm 5.567)$ years, and females constituted $(66.7 \%)$ of this group. Poor medication compliance was the commonest risk factor associated with DKA, and this relationship was found significant (P-value 0.003). Out of the total DKA sufferers, (28) patients experienced recurrent or multiple DKA episodes, and those had higher HbA1C levels $(11.7 \pm 2.9$ Vs $12.4 \pm$ 3.3). Patients who had multiple DKA episodes reported less visits to the diabetes educator when compared to the single DKA group. (On average (9) Vs (11) per year).

Conclusion: DKA was found relatively uncommon among our DM1 patients, and usually seen in young females with poor medication compliance. More comprehensive education on DKA is needed to protect against life threatening complications.
\end{abstract}

Key-words: IDDM -Diabetic Ketoacidosis (DKA) -Diabetic Education

\section{INTRODUCTION}

Diabetes is a recognizable and major health burden in Saudi Arabia. In fact, more than 3.4 million citizens were diagnosed with diabetes in the year $2015^{(1,2)}$. Many factors have been implicated in the etiology of the disease, and all are directly related to disorders of insulin production, action or both. The resulting failure of glucose uptake by human cells leading to hyperglycemia, can start a series of serious metabolic changes and chronic organ dysfunctions. Different categories of diabetes were identified: Type (1), Type (2), gestational diabetes, plus forms resulting from other conditions such as pancreatitis or consumptions of large doses of exogenous steroids. As one of the most prevalent chronic conditions in our region, all these types of diabetes are encountered in Saudi Arabia.

The Insulin Dependent Diabetes Mellitus (IDDM) or Diabetes Mellitus type 1 (DM1) is an autoimmune disease which targets insulinproducing, pancreatic $\beta$ cells, and although its incidence is variable across different populations, it is predominant among younger age groups ${ }^{(3,4)}$. In Saudi Arabia, a research conducted between the years 2001-2007 reported DM1 incidence to be 109.5 per 100,000 child and adolescent ${ }^{(5)}$.

One of the major life-threatening complications of DM1is Diabetic Ketoacidosis (DKA). DKA is an acute metabolic state characterized by massive elevation of glucose and ketones blood levels (6). Patients mostly present with polyuria, polydipsia, vomiting, and abdominal pain. The resultant dehydration and metabolic acidosis can result in tachycardia, hyperventilation, confusion and in severe forms coma and death ${ }^{(}{ }^{(}$. DKA can be the first presentation for non-diagnosed DM1patients, and one third of DM1 patients' admissions to the Intensive Care Units are due to severe DKA, with a (13\%) inhospital mortality rate ${ }^{(8)}$. Globally, researchers have studied factors attributed to recurrent DKA onsets, such as: medication non-compliance, infections, mental health issues and stress, and even other demographic factors such as age and gender ${ }^{(7)}$, and despite classifying Saudi Arabia among the top three countries with highest incidence of DKA presentations in the region, a limited number of studies focused on describing and quantifying DKA occurrence. This study aims to estimate DKA incidence among DM1 patients at King Abdulaziz Medical City (KAMC), describe episodes' characteristics including recurrence, and identify possible risk factors.

\section{PATIENTS AND METHODS}

The study was a two months' retrospective review, for DM1 patients registered and followed at King Abdulaziz Medical City (KAMC) in Jeddah, between the first of January 2010 and until the end of June 2016. We reviewed electronic files of all patients admitted between the ages of 14-40, and 
only extracted data for those who presented with DKA during the designated six and half years' study period. DM1 patients with who were younger than 14 and older than 40, who suffered from chronic illnesses and/or were on corticosteroids were excluded. Ethical approval was obtained from the Research Ethics Review Board at KAMC along with the other necessary administrative approvals.

We used a standardized data extraction sheet to collect relevant patients' information. The latter included: General demographics such as; date of birth, gender and weight, DM1 clinical profile including; date of DM1 diagnosis, their concurrent insulin regimen, number of follow-up visits and meetings with the diabetes educator during the study period and patient compliance reflected by HbA1C. It indicates the level of average blood sugar over the time of approximately three months. Lastly, we focused on the Past medical history of DKA; the number of DKA episodes, severity of each episode and the possible risk factors.

Following international guidelines and the standard DKA management protocol used at KMAC, a DKA episode was defined as; "Serum glucose level greater than $250 \mathrm{mg}$ per $\mathrm{dL}$, a $\mathrm{pH}$ less than 7.3, a serum bicarbonate level less than $18 \mathrm{mEq}$ per $\mathrm{L}$, an elevated serum ketone level with dehydration". A "recurrent DKA" _status was used to describe patients who had more than one DKA episode during the study period. To assess the severity of each DKA episode, we adopted a biochemical classification that was previously applied with DM1 patients. It is based on both the intensity of metabolic acidosis, (determined by level of arterial $\mathrm{pH}$, serum bicarbonate, urine ketone, serum ketone and anion gap), plus the mental status at the time of presentation. Further details on the rationale and application of this classification was described elsewhere ${ }^{(6)}$. According to these parameters, a DKA episode was classified as "mild, moderate, or severe". We documented $\mathrm{HbA1C}$ levels that were closest to the latest DKA episode within the previous three months.

Extracted data was coded, crosschecked and then entered on daily basis. Statistical analysis was performed using a statistical package for social sciences (SPSS) version 20 (SPSS Inc., Chicago, IL, USA).

Descriptive analysis was used to determine the frequency of study variables. To establish a relationship between categorical variables, we used chi-square test. A P-value of $(<0.05)$ was considered statistically significant. It must be acknowledged that the original scope of the study was designed to include other demographic variables, however the pilot search revealed minimal reporting on this information, and thus we had to limit our search.

\section{RESULTS}

Three hundred and fifty DM1 patients were included in this study, and 72 of them presented with DKA during our study period. They represented $(20.6 \%)$ of the total DM1 patients followed at KMAC. They have lived with the disease for an average of 9.89 \pm 6.47 years. Their mean age was $22.82 \pm 5.567$ years, with females constituting around two third of this group $(66.7 \%)$, and the remaining (33.3\%) were males. For their treatment regimens, most patients were on a " 4 times per day" injectable regimen (86.1\%), few were on the conventional "twice daily" regimen $(9.7 \%)$, and even less were using pumps (4.2\%).

Together, these 72 patients experienced a total of 141 DKA episodes during the six and a half years, with an average annual DKA onset of $6.2 \%$ among all DM1 patients in KMAC. When disaggregated per year, the annual DKA incidence was $4.85 \%$ in $2010,4.28 \%$ in $2011,2.85 \%$ in 2012 , $6.85 \%$ in $2013,8 \%$ in $2014,10.57 \%$ in 2015 , and $2.85 \%$ for the year 2016 . Regarding the severity of these episodes, $61(43.3 \%)$ episodes were classified as mild, 41(29.1\%) as moderate and 39(27.6\%) episodes were severe, respectively.

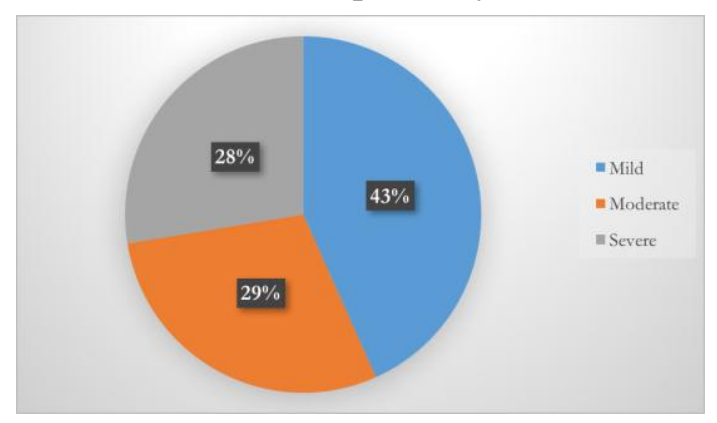

Figure (1): Severity of DKA episodes

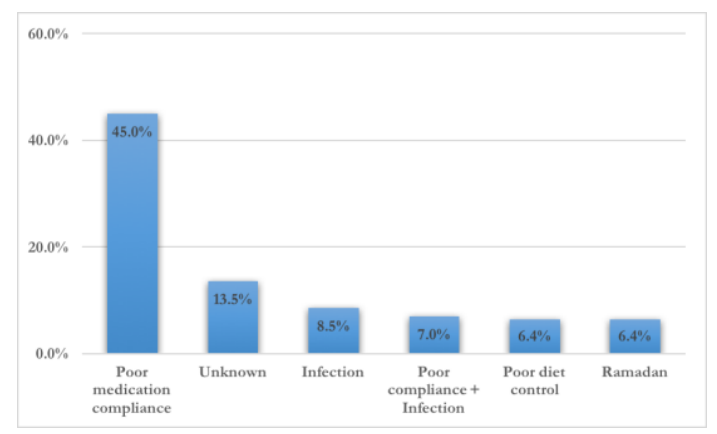

Figure (2): Factors associated with an onset of DKA. 
(Figure1) Almost one third of these patients were admitted to the Intensive Care Unit (ICU) at least once. Yet, only 2 patients developed complications and the remaining patients resolved completely. For $10.6 \%$ of episodes, DKA was the patient's first reported presentation. Among the possible factors associated with DKA, poor medication compliance was the commonest one reported (45\%), followed by infections $(8.5 \%)$ and then poor diet control (6.4\%) (Figure2) A significant difference was detected, indicating an association between poor medication compliance, and increased DKAs onset (P-value 0.003).

Forty-four patients of the total DKA suffers reported a single DKA episode only, while the remaining (28) patients experienced recurrent or multiple DKA episodes during the

study period. For the latter group, 14 patients experienced two episodes, 5 suffered three DKAs, and for 9 patients more than three episodes were documented in the study period. Both groups were within the same age group $(22.9 \pm 5.7 \mathrm{Vs}$ $22.8 \pm 5.5$ ). As for gender, they were constituted similarly, with a slightly higher female proportion in the multiple DKAs group (65.9\% Vs 67.9\%). Regarding treatment regimen, the majorities of both groups were on "4 times daily" injectable insulin, however the proportion of these users and who experienced multiple DKAs was higher (81.8\% Vs $92.9 \%)$. Although both groups exhibited relatively poor compliance, as per HbA1C levels, patients who reported a single DKA episode showed better compliance when compared to the other group $(11.7 \pm 2.9$ Vs $12.4 \pm 3.3)$ (Table1).

Table (1): Characteristics of patients presenting with DKA during study period.

\begin{tabular}{|l|l|l|}
\hline \multirow{2}{*}{ Characteristics } & \multicolumn{2}{|c|}{ Patients per DKA Episodes } \\
\cline { 2 - 3 } & $\begin{array}{c}\text { Single DKA } \\
\text { episode (44) }\end{array}$ & $\begin{array}{c}\text { Multiple DKA } \\
\text { episodes (28) }\end{array}$ \\
\hline Age (Yrs) & $22.9 \pm 5.7$ & $22.8 \pm 5.5$ \\
\hline HBA1C & $11.7 \pm 2.9$ & $12.4 \pm 3.3$ \\
\hline Gender & $15(34.1 \%)$ & $9(32.1 \%)$ \\
\hline Male & $29(65.9 \%)$ & $19(67.9 \%)$ \\
\hline Female & $2(7.1 \%)$ \\
\hline Treatment regimen & \multicolumn{2}{|l}{} \\
\hline Conventional Oral & $5(11.4 \%)$ & $26(92.9 \%)$ \\
\hline Injectable Insulin & $36(81.8 \%)$ & $0(0.00 \%)$ \\
\hline Insulin Pump & $3(6.8 \%)$ &
\end{tabular}

All 72 patients underwent regular followup and counselling sessions, with both attending physicians and diabetic educators. With respect to clinic visits, and for the total of the study period, single DKA sufferers reported an average of 4 visits per patient, while a multiple DKA sufferer came to these clinics about 5 times throughout the same period. On the other hand, each patient who reported multiple DKA episodes, documented an average of 9 visits to the diabetes educator during the study period, whereas those who only had a single DKA visited the educators 11 times.

\section{DISCUSSION}

This study estimated the annual DKA incidence among DM1 patients attending KAMC facilities in Jeddah. It also explored on risk factors possibly associated with recurrent DKA admissions. For our group, the frequency of DKA was found to be $6.2 \%$. This reassuring rate is considered relatively law when compared to higher incidences elsewhere, both regionally and nationally. A systematic review exploring DKA epidemiology in twelve Arab countries revealed an overall rate of $46.7 \%$ among DM1 patients ${ }^{(10)}$. Rates started at $17 \%$ and reached as high as $100 \%$ in Algeria ${ }^{(11)}$. and Morocco ${ }^{(12)}$. On the other hand, rates reported for other facilities across Saudi Arabia were also much higher and quite variable, ranging between $56 \%^{(13)}$ in the central region to reach $80 \%$ in the western region ${ }^{(10,14)}$.

Global studies documented more frequent DKA rates among children, with more than $80 \%$ presenting to the hospital for first time with DKA ${ }^{(15,16)}$. Similar conclusions were drawn from studies conducted in Saudi Arabia ${ }^{(13,14)}$. However, most of our group were young youth, slightly older than the usual DM1 cases. The latter can explain the low DKA rates reported in KMAC. Older patients are expected to be more aware about their condition, the common risk factors and complications and means to protect against them.

Regarding the recurrence of DKA episodes, 28 patients experienced multiple DKAs constituting $(8 \%)$ of the total DM1 patients in KMAC, and 39\% of the total DKA suffers. Accordingly, DKA itself is not a common presentation for DM1 patients in KMAC, yet, it tends to reoccur for the same patients. The latter highlights the need to closely follow up and support these patients. Another notable observation was the disaggregated DKA rates by gender, as more females suffered multiple DKAs during the study period. This come in agreement with some literature suggesting that females are more prone to 
recurrent DKA when compared to males ${ }^{(13,17,18)}$. Young girls are very self-aware about body image, and are highly affected with their surroundings. They may aim to maintain or lose weight, then skip insulin doses, and thus increasing the risk of DKA. It is important to take that into consideration when delivering care to these girls, especially with nutritional and psychological support.

The major precipitating factor for DKA was poor medication compliance $(45 \%)$ in our sample. The literature identified several factors associated with DKA, including poor adherence to medication, uncontrolled diet, disrupted family status and lack of accessibility of health services $(13,17,18,19,20,21)$. Yet, a larger number of studies described insulin non-adherence or even discontinuation as commonest cause of DKA recurrence ${ }^{(13,17,18,19,20)}$. This emphasizes the value of increasing awareness on DKA and its risk factors, and more aggressively on medication use and the importance of compliance. HbA1C is the most widely used indicator to assess patient's glycemic control, for both diet and medication compliance. It is a major predictor for DKA occurrence. Elevated levels can indicate higher risk of developing diabetic complications, ${ }^{(9)}$ and in fact patients with $\mathrm{HbA1C}$ levels $\geq 10.0 \%$ have higher risks of experiencing DKA specifically ${ }^{(22)}$. In our study, among all DM1 patients, those with the longer disease history showed better HBA1C levels, and thus better metabolic control and less DKA episodes. Patients may become more aware about their disease with time, and tend to better maintain blood sugar levels. This again highlights the importance of raising patients' awareness and diabetes education, including measures considered preventive against DKA.

We also noted a difference in $\mathrm{HbA} 1 \mathrm{C}$ levels between single and multiple DKA sufferers, favoring better control in the single DKA sufferers, yet and because $\mathrm{HbA1C}$ was not

measured with every DKA episode, we could not test the statistical significance of this relationship.

When exploring on follow-up modalities, on average multiple DKA suffers reported one extra clinic visit when compared to single DKA suffers, probably since multiple DKA admissions demands extended follow-up with the attending physician. As those who suffered more than two DKA episodes are expected to demand further intensified care, and more clinic visits, we aimed to test the relationship between the number of visits and the numbers of DKAs. Unfortunately, within records, reported $\mathrm{HbA} 1 \mathrm{C}$ levels were not related to different DKA episodes. On the other hand, single DKA sufferers had more visits to the diabetes educators when compared to multiple DKA sufferers, suggesting the protective and supportive role of these visits on DM1 patients, and while global studies acknowledged the added value of comprehensive and multidisciplinary diabetes educators, ${ }^{(23,24)}$ specifically on glycemic control and minimizing complications, (25) a limited number of studies focused on that in Saudi Arabia. More research is needed to assess the impact of diabetes education, and determine its relevance to patient's need.

\section{CONCLUSION}

DKA was found relatively uncommon among our DM1 patients, usually seen in young females with poor medication compliance, and with no residual complications. More dedicated and comprehensive education on DM and DKA is a must to control it recurrence and other life threatening complications.

\section{REFERENCES}

1. International Diabetes Federation (2013): IDF Diabetes.Atlas Sixth Edition. https:// www.idf.org/e-library/epidemiology.../diabetesatlas/ 19-atlas-6th-edition.htm

2. Alqurashi KA, Aljabri KS, Bokhari SA (2011): Prevalence of diabetes mellitus in a Saudi community. Annals of Saudi Medicine, 31:19-23.

3. Atkinson M, Eisenbarth G (2001): Type 1 diabetes: new perspectives on disease pathogenesis and treatment. The Lancet, 358: 221-229.

4. Maahs $\mathrm{D}$, West $\mathrm{N}$, Lawrence $\mathrm{J}$ et al. (2010): Epidemiology of type 1 diabetes. Endocrinology and Metabolism Clinics, 39: 481-497.

5. Al-Herbish A, El-Mouzan M, Al-Salloum A et al. (2008): Prevalence of type 1 diabetes mellitus in Saudi Arabian children and adolescents. Saudi medical journal,29:1285-8. 
6. Kitabchi A, Umpierrez G, Miles J, Fisher J (2009): Hyperglycemic crises in adult patients with diabetes. Diabetes care,32:1335-43.

7. Randall L, Begovic J, Hudson M et al. (2011): Recurrent diabetic ketoacidosis in inner-city minority patients: behavioral, socioeconomic, and psychosocial factors. Diabetes care, 34:18916.

8. Efstathiou $S$, Tsiakou A, Tsioulos $D$ et al. (2002): A mortality prediction model in diabetic ketoacidosis. Clinical endocrinology,57:595-601.

9. World Health Organization. (2011): Use of glycated haemoglobin (HbA1c) in the diagnosis of diabetes mellitus. http:// www. who. int/ diabetes/ publications/ diagnosis_diabetes2011/ en/

10. Zayed H (2016): Epidemiology of diabetic ketoacidosis in Arab patients with type 1 diabetes: a systematic review. International journal of clinical practice,70:186-195.

11. Raache $\mathrm{R}$, Belanteur $\mathrm{K}$, Amroun $\mathrm{H}$ et al. (2012): Association of major histocompatibility complex class 1 chain-related gene a dimorphism with type 1 diabetes and latent autoimmune diabetes in adults in the Algerian population. Clinical and Vaccine Immunology, 19: 557-61.

12. Izaabel $H$, Garchon $H$, Beaurain $G$ et al. (1996): Distribution of HLA class II alleles and haplotypes in insulin-dependent Moroccan diabetics. Human immunology, 49: 137-43.

13. Al-Hayek A, Robert A, Braham R, Turki A, Al-Sabaan F (2015): Frequency and associated risk factors of recurrent diabetic ketoacidosis among Saudi adolescents with type 1 diabetes mellitus. Saudi Medical Journal,36:216-20.

14. Alanani K, Mohamed N, Alsulaimani A (2013): Epidemiological pattern of newly diagnosed children with type 1 diabetes mellitus, Taif, Saudi Arabia. The Scientific World Journal, 279:421569

15. Fritsch $M$, Rosenbauer J, Schober $\mathbf{E}$ et al. (2011): Predictors of diabetic ketoacidosis in children and adolescents with type 1 diabetes. Experience from a large multicentre database. Pediatric diabetes, 12:307-312.
16. Usher-Smith J, Thompson M, Ercole A, Walter F (2012): Variation between countries in the frequency of diabetic ketoacidosis at first presentation of type 1 diabetes in children: a systematic review. Diabetologia,55:2878-2894.

17. Karges B, Rosenbauer J, Holterhus $P$ et al. (2015): Hospital admission for diabetic ketoacidosis or severe hypoglycemia in 31,330 young patients with type 1 diabetes. European journal of endocrinology, 173:341-50.

18. Rewers A, Chase H, Mackenzie T et al. (2002): Predictors of acute complications in children with type 1 diabetes. Jama., 287: 2511-2518.

19. Lohiya S, Kreisberg R, Lohiya V (2013): Recurrent diabetic ketoacidosis in two community teaching hospitals. Endocrine Practice, 19: 829-833.

20. Morris A, Boyle D, McMahon A et al. (1997): Adherence to insulin treatment, glycaemic control, and ketoacidosis in insulin-dependent diabetes mellitus. The Lancet,350:1505-1510.

21. Hanas R, Lindgren F, Lindblad B (2009): A 2- yr national population study of pediatric ketoacidosis in Sweden: predisposing conditions and insulin pump use. Pediatric diabetes, 10:3337.

22. Weinstock R, Xing D, Maahs D et al. (2013): Severe hypoglycemia and diabetic ketoacidosis in adults with type 1 diabetes: results from the T1D Exchange clinic registry. The Journal of Clinical Endocrinology \& Metabolism, 98:34113419.

23. Dudley J (1980): The diabetes educator's role in teaching the diabetic patient. Diabetes Care, 3:127-133.

24. Davis E (1990): Role of the diabetes nurse educator in improving patient education. The Diabetes Educator, 16: 36-38.

25. Delahanty L, Halford B (1993): The role of diet behaviors in achieving improved glycemic control in intensively treated patients in the Diabetes Control and Complications Trial. Diabetes care, 16:1453-1458. 